\title{
Relative Angle Estimation of an Interferer in a Diffusion-based Molecular Communication System
}

\author{
Eric Regonesi \\ Mariangela Rapisarda \\ DEIB, Politecnico di Milano \\ Milan, Italy \\ eric.regonesi@polimi.it \\ mariangela.rapisarda@polimi.it
}

\author{
Maurizio Magarini \\ DEIB, Politecnico di Milano \\ CNIT \\ Milan, Italy \\ maurizio.magarini@polimi.it
}

\author{
Marco Ferrari \\ CNR-IEIIT \\ DEIB, Politecnico di Milano \\ Milan, Italy \\ marco.ferrari@ieiit.cnr.it
}

\begin{abstract}
The design of molecular communication systems over a diffusive channel has been extensively studied under the hypothesis of a point-wise transmitter and one receiving cell that absorbs molecules from the environment. Recent works have extended this scenario by including also the effect of one, or more, interfering cells that introduce a perturbation in the number of molecules absorbed by the target receiving cell. In this paper we exploit such a perturbation to estimate the relative angle under which the receiver sees the interferer with respect to the transmitter. The mean-squared error of the relative angle estimation is reported for different distances between interferer and receiver. As a main result, we show that the interfering cell introduces two effects, namely "blocking" and "shadowing", that strongly affect the angle assessment. Simulation results are supported by the derivation of an analytical model that is able to make a good prediction of the average number of molecules absorbed by the target receiver as a function of the position of the interferer. Our numerical results show that, for the selected hypotheses, the best performance for the angle estimation is achieved when it is around $30^{\circ}$.
\end{abstract}

\section{KEYWORDS}

Molecular communication, angle estimation, interfering cell.

\section{ACM Reference Format:}

Eric Regonesi, Mariangela Rapisarda, Maurizio Magarini, and Marco Ferrari. 2020. Relative Angle Estimation of an Interferer in a Diffusion-based Molecular Communication System. In Proceedings of 7th ACM International Conference on Nanoscale Computing and Communication (College Park '20). ACM, New York, NY, USA, 7 pages. https://doi.org/10.1145/nnnnnnn.nnnnnnn

\section{INTRODUCTION}

A more and more widespread development of synthetic biology, along with advances in nanotechnology and medicine, is pushing researchers to investigate the Nature (e.g. both animal and plant tissues, or bacteria) under a different light. Cells communicate

Permission to make digital or hard copies of all or part of this work for personal or classroom use is granted without fee provided that copies are not made or distributed for profit or commercial advantage and that copies bear this notice and the full citation on the first page. Copyrights for components of this work owned by others than ACM must be honored. Abstracting with credit is permitted. To copy otherwise, or republish, to post on servers or to redistribute to lists, requires prior specific permission and/or a fee. Request permissions from permissions@acm.org.

College Park '20, September 23-25, 2020, College Park, MD

(C) 2020 Association for Computing Machinery.

ACM ISBN 978-x-xxxx-xxxx-x/YY/MM...\$15.00

https://doi.org/10.1145/nnnnnnn.nnnnnnn each other by exchanging particular substances. Some examples of communication within a human body are the release by the endocrine system of hormones, which propagate in bloodstream, or the release of neurotransmitters in the synapses connecting neurons, which enable a communication between them [1].

A group of cells exchanging substances can be studied as a communication system. The characterization and exploitation of this kind of systems are the core topics of an emerging line of research: molecular communication (MC). In such systems, one or more cells emitting substances represent the transmitters, which encode the information in the concentration and/or in the type of sent molecules. Likewise, the cells that absorb the molecules correspond to the receivers. The surrounding space is the channel, whose effect has to be characterized in different scenarios. MC is a promising research field, which might give advantage in many sectors, e.g. in medicine, to build nanomachines able to administer drugs to the affected area of a body, and to limit the impact of such drugs on the other organs [3] or in aerospace engineering, for the localization of sunk aeroplanes through the creation of novel black boxes releasing also signalling substances [11], just to cite a few.

An extensive number of works focus on MC systems defined by a single transmitting and single receiving cells, some of which progressively extend the case-study to a more complex scenario. The actual low performance of diffusive MC systems has suggested the adoption of Multiple-Input Multiple-Output (MIMO) schemes $[7,8]$. The price to be paid for an increased channel capacity is the intrinsic Inter-Link Interference, i.e. the absorption of molecules targeted to a specific cell by the other receivers. Here, we want to exploit the same disturbance for localization purposes.

This work focuses on the simplified MC scenario defined in [9], which consists of a transmitter, a "target" receiver, and an interferer. The interfering cell absorbs part of the diffused molecules. As a main contribution, we propose an approach to estimate the angle under which the receiving cell sees the interfering cell with respect to the direction defined by the segment that joins the transmitter and the target receiver. Although there are many works that focus on the problem of estimating the distance between a transmitting and a receiving cell $[5,10,15]$, to the best of authors' knowledge no one has considered the possibility of estimating the direction under which an interfering cell at known distance is seen by the receiver.

The proposed approach exploits the perturbation induced by the interfering cell on the number of molecules received by the target receiver under the hypotheses of knowing the distances between transmitting and target receiving cells and between the target and 


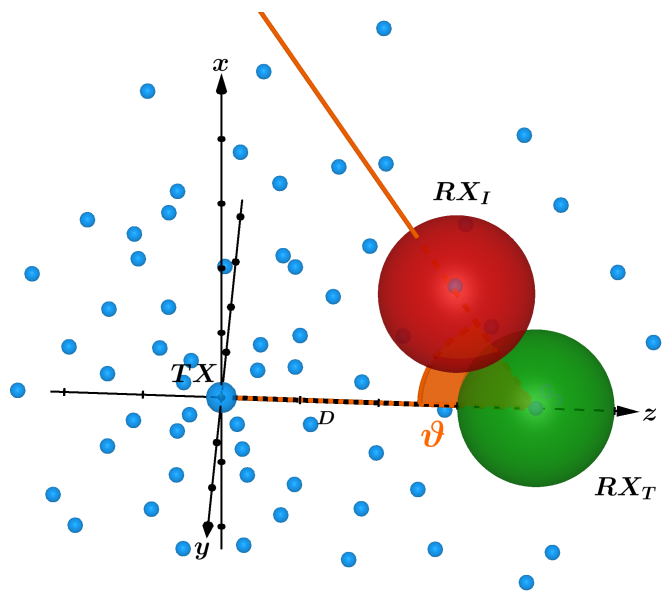

Figure 1: MC system under study: $T X$, transmitting cell, $R_{T}$ target receiver, and $\mathrm{RX}_{\mathrm{I}}$ interfering cell.

the interfering cell. We show that, depending on the closeness of the interfering cell to the transmitter or to the target receiver, the best estimation of the relative angle is achieved when it is around $30^{\circ}$.

It is worth observing that the perturbation introduced by the presence of an absorbing interferer in the environment was already considered in [9] to evaluate the degradation on the Bit Error Rate (BER) and on the information capacity by using a simulation approach. In [2] a first attempt is done to derive an analytical expression of the channel impulse response between transmitter and receiver that includes the effect of the perturbation introduced by the interferer. The formula accounts for the presence of the interferer by introducing a scaling factor in the expression that gives the number of molecules absorbed by the receiver as a function of time $t$ with respect to the case where it is absent. The scaling factor is expressed as a function of some adjustment coefficients that depend on the relative angle under which the point-wise transmitter sees the two absorbing receivers, and on the distance of the interferer from the transmitter. The adjusting coefficients are empirically obtained by a curve fitting approach for each relative angle, transmitter-interferer distance, and transmitter-receiver distance. Therefore, if we wanted to define an estimator for the relative angle that relies on such a scaled channel impulse response, this would have the disadvantage of requiring to run simulations for each scenario. In place of trying to apply such a model, this paper presents an alternative analytical model that does not require the fitting of simulation results. The analytical model provides a tool to estimate the average number of absorbed molecules versus $t$ when there is an interferer.

The paper is organized as follows. Section 2 describes the scenario with two absorbing receivers that is implemented in our work and its contextualization in a real biological setting. Numerical results are shown in Sec. 3 together with the proposed angle estimation approach and its performance in terms of mean-squared error (MSE). The proposed analytical models are described in Sec. 4.
Finally, Sec. 5 summarizes the results and proposes some further directions to extend the current work.

\section{INVESTIGATED MC SCENARIO}

A pictorial description of the scenario under investigation is shown in Fig. 1. A point-wise transmitting cell (TX) emits impulsively $N_{T}$ molecules in a fluid. A spherical receiving cell $\mathrm{RX}_{\mathrm{T}}$ with radius $\mathrm{R}$ is located at distance $D$ from it. An identical interfering cell $\mathrm{RX}_{\mathrm{I}}$ is placed at a distance $d$ from $\mathrm{RX}_{\mathrm{T}}$ (center-to-center distance). Both receivers are supposed to be fully absorbing, i.e. the absorption probability of the receivers is set to be $p_{a b s}=1$. It follows that when a molecule $m$ is at a distance $d_{m} \leq R$ from the center of any receiver, it is absorbed and removed from the environment [13].

Once released in the environment, each molecule, assumed to be dimensionless, moves in the fluid medium through a diffusion process characterized by a low Reynolds number [4]. Thus, the generic molecule coordinates $\left(x_{m}(t), y_{m}(t), z_{m}(t)\right)$ at time $t$ are updated at the time instant $t+\Delta t$ according to a random walk:

$$
\begin{aligned}
& x_{m}(t+\Delta t)=x_{m}(t)+\zeta_{1} \sqrt{2 c_{d} \Delta t}, \\
& y_{m}(t+\Delta t)=y_{m}(t)+\zeta_{2} \sqrt{2 c_{d} \Delta t}, \\
& z_{m}(t+\Delta t)=z_{m}(t)+\zeta_{3} \sqrt{2 c_{d} \Delta t},
\end{aligned}
$$

where $c_{d}$ is the diffusion coefficient, $\Delta t$ is the time step, $\zeta_{1}, \zeta_{2}$, and $\zeta_{3}$ are Gaussian independent random variables, with mean $\bar{\zeta}_{i}=0$ and variance $\sigma_{\zeta_{i}}^{2}=1(i=1,2,3)$.

The system parameters are listed in Table 1 . The dimensions and shapes of the receivers are compatible with bacteria with coccus cell morphology (e.g. Staphylococcus species, Streptococcus species, etc.), round-shaped bacteria whose diameter can vary in the range $[0.25,1] \mu \mathrm{m}[14]$. The case study of this paper can be applied to model the quorum sensing phenomenon described in many bacteria, by which they can perceive the number of microorganisms necessary to activate the expression of factors needed, for instance, to colonize a host (in case of pathogenic bacteria) [12], or to create an aggregation forming a biofilm [6].

Table 1: System parameters and their values

\begin{tabular}{|c|c|c|}
\hline Definition & Parameter & Value \\
\hline Number of released & $N_{T}$ & $1 \cdot 10^{4}$ \\
molecules & & \\
Diffusion coefficient & $c_{d}$ & $79.4 \mu \mathrm{m}^{2} / \mathrm{s}$ \\
Distance $\mathrm{TX}-\mathrm{RX}_{\mathrm{T}}$ & $D$ & $\in[4,10] \mu \mathrm{m}$ \\
Distance $\mathrm{RX}_{\mathrm{T}}-\mathrm{RX}_{\mathrm{I}}$ & $d$ & Variable \\
Coordinates of TX & $\left(x_{T}, y_{T}, z_{T}\right)$ & $(0,0,0)$ \\
Coordinates of $\mathrm{RX}_{\mathrm{T}}$ & $\left(x_{R}, y_{R}, z_{R}\right)$ & $(0,0, D)$ \\
Coordinates of $\mathrm{RX}_{\mathrm{I}}$ & $\left(x_{I}, y_{I}, z_{I}\right)$ & $\left(x_{I}, 0, z_{I}\right), \mathrm{see}(2)$ \\
Receiver radius & $R$ & $\in[0.25,1] \mu \mathrm{m}$ \\
Real angle & $\vartheta$ & $\in\left[0^{\circ}, 180^{\circ}\right]$ \\
Estimated angle & $\hat{\vartheta}$ & $\in\left[0^{\circ}, 180^{\circ}\right]$ \\
Time step & $\Delta t$ & $1 \cdot 10^{-4} \mathrm{~s}$ \\
Simulation time & $T_{\text {sim }}$ & $0.5 \mathrm{~s}$ \\
\hline
\end{tabular}


Without loss of generality, we assume that the TX lies in the origin, $\mathrm{RX}_{\mathrm{T}}$ has its center at a distance $D$ from TX along the z-axis while that of $\mathrm{RX}_{\mathrm{I}}$ is placed somewhere in the $x z$ plane. Both $D$ and $d$ are known by hypothesis, even if we are aware that the estimation of the distances could be impaired by the presence of the interfering cell. The coordinates of $\mathrm{RX}_{\mathrm{I}}$ and $\mathrm{RX}_{\mathrm{T}}$ are related as

$$
\begin{aligned}
& x_{I}=x_{R}+d \cdot \sin \vartheta \\
& z_{I}=z_{R}-d \cdot \cos \vartheta
\end{aligned}
$$

It is worth noting that the obvious ambiguity related to the threedimensional symmetry of the problem does not allow us to identify the position of the interfering cell in the plane perpendicular to the segment joining TX and $\mathrm{RX}_{\mathrm{T}}$ on which the center of $\mathrm{RX}_{\mathrm{I}}$ lies.

In order to estimate the relative angle under which the receiving cell sees the interferer, the following variables can be measured:

(a) number of molecules absorbed at the target receiver $N_{R}$, either in absolute value or as a difference with respect to the case without interferer. The angle estimation is performed after defining thresholds on the mean number of received molecules in the form of look-up tables or through analytical regression on the same quantity;

(b) variation of the distribution of molecules concentration with respect to the absence of the interfering cell in each receptor, or group of neighbouring receptors, that is located on the surface of the receiving cell;

(c) modification at $\mathrm{RX}_{\mathrm{T}}$ of the channel impulse response with or without $\mathrm{RX}_{\mathrm{I}}$.

In this paper we consider the measure of the number of molecules as defined in (a) since it provides the most intuitive variable to estimate $\vartheta$, i.e. based on the simple observation of the number of received molecules. The usage of molecules concentration in (b) appears to be more complex since it would require the cell to be aware of the entrance position of the molecules. Finally, the channel impulse response modification in (c) exploits the information associated with the evolution in time, in contrast to the integral value in (a), and, therefore, it is left to future work.

\section{NUMERICAL RESULTS AND RELATIVE ANGLE ESTIMATION METHOD}

In order to count the number of absorbed molecules we built a numerical simulator. In the following we show simulation results obtained for different values of the parameters that define the configuration of the scenario. In the current literature, the transmission system with two receivers has been studied in terms of channel capacity and BER [9]. For localization of the interferer position via the angle estimation method we need to know the expected number of received molecules at the target receiving cell. The intrinsic dependence on tabulated coefficients in [2] does not allow us to perform predictions of the received molecules for the considered scenario. Thus, we have split our investigation into two steps: i) estimation of the mean number of received molecules $\bar{N}_{R}$ and ii) localization of the interfering cell.

First, we found $\bar{N}_{R}$ for different values of the receiver cell radius $R$ and of the distances $D$ and $d$, both in the presence and in the absence of the interferer. Then, we considered a specific scenario suitable to perform the angle estimation, i.e. we have fixed the values
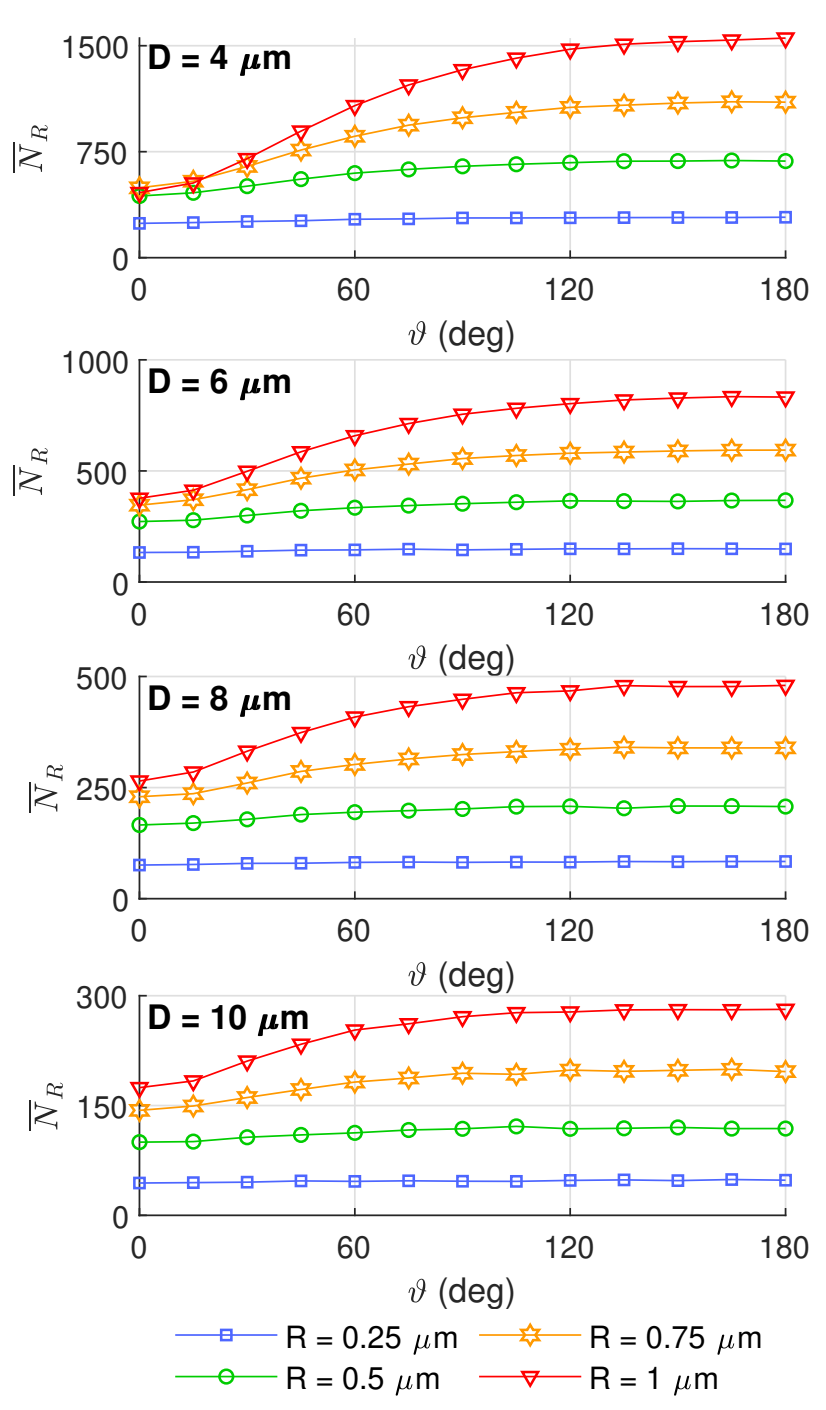

Figure 2: Mean number of absorbed molecules at $\mathbf{R} \mathbf{X}_{\mathrm{T}}$, for different distances and radii.

of $R, D$ and $d$. All the presented results were obtained with a simulation time of $T_{\text {sim }}=0.5 \mathrm{~s}$, which is a good compromise between the numerical speed performance and the accuracy of the results. The gap between different $\vartheta$ was set to $15^{\circ}$, whereas the maximum angle considered was $180^{\circ}$. Numerical values were obtained for $M=200$ independent trials, where not differently specified.

\subsection{Impact of $R$ on the mean number of absorbed molecules}

Initially, we investigated the impact of the cells' radius on the mean number of absorbed molecules as a function of the relative angle $\vartheta$. Figure 2 reports the mean number of molecules $\bar{N}_{R}$ absorbed by $\mathrm{RX}_{\mathrm{T}}$ in the presence of $\mathrm{RX}_{\mathrm{I}}$ for different values of the radius $R$. Simulations were performed for some values of $D$ by keeping $d=D / 2$, 


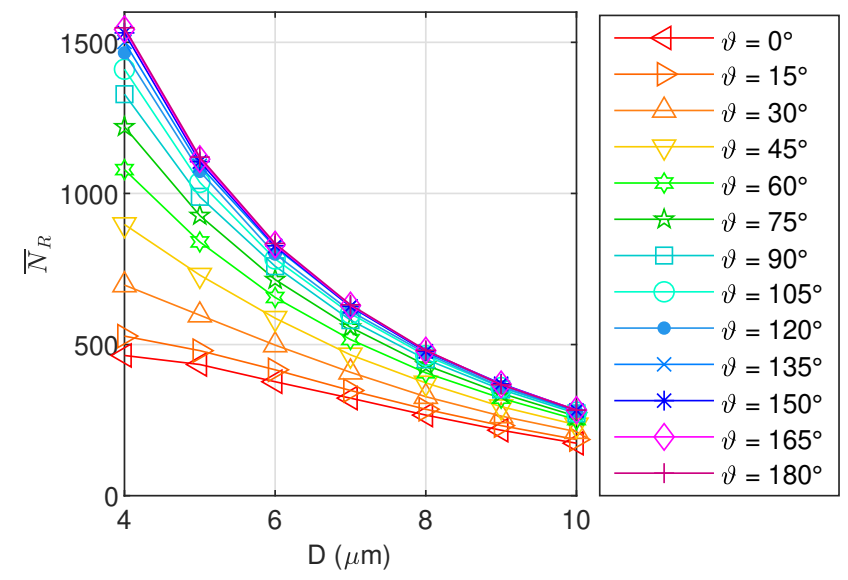

Figure 3: Mean number of absorbed molecules at $R \mathbf{X}_{\mathrm{T}}$ versus $D$, for $R=1 \mu \mathrm{m}$.

which is exactly half of the distance between transmitting and receiving cell when $\vartheta=0^{\circ}$. The figure shows that for the considered values of $D$ a sensible variation in the average number of molecules as a function of $\vartheta$ can be observed only for $R=1 \mu \mathrm{m}$ when $\vartheta \leq 120^{\circ}$. For $R=0.75 \mu \mathrm{m}$ a significant variation can be observed only at low values of $D$, i.e. when the receiver is close to the transmitter. For $R=0.25 \mu \mathrm{m}$ and $R=0.5 \mu \mathrm{m}$ the position of the interferer would be hardly detected, since its presence weakly influences an already low absorption. This is somehow expected, since smaller cells capture less molecules. From these results it is possible to conclude that a good discrimination of the relative angle of the interferer is possible at large values of the radius and that a better performance is obtained when the distance between transmitter and intended receiver is low.

\subsection{Effect of $D$ on the mean number of absorbed molecules}

For this case we set $R=1 \mu \mathrm{m}$, which is the most sensitive value of receiver radius among the ones presented in 3.1, always keeping $d=D / 2$. Figure 3 explicitizes on the $x$-axis the variation of the number of absorbed molecules versus the distance $D$ for different values of $\vartheta$. We can observe that, whatever the angle of the interfering cell is, the number of absorbed molecules decreases along with the increase of the transmitter-target receiver distance. The most sensitive distance is $D=4 \mu \mathrm{m}$, which corresponds to $d=2 \mu \mathrm{m}$, i.e. the limit condition for which the two receivers are in contact. It is also observed that the spread among the curves reduces towards $D=10 \mu \mathrm{m}$ but without crossings, a good condition for the introduction of thresholds in the angle estimation.

The behavior of $\bar{N}_{R}$ versus $\vartheta$ is shown in Fig. 4, which is the dual of Fig. 3, for different values of $D$. The effect of the interferer position is more disruptive on $\bar{N}_{R}$ when it is on the same line of TX and $\operatorname{RX}_{\mathrm{T}}\left(\vartheta=0^{\circ}\right)$, which is likely caused by the absorption of a large number of molecules emitted by TX. As $\vartheta$ increases, the "shadowing" effect of $\mathrm{RX}_{\mathrm{I}}$ decreases. At $\vartheta=180^{\circ}$ the influence of

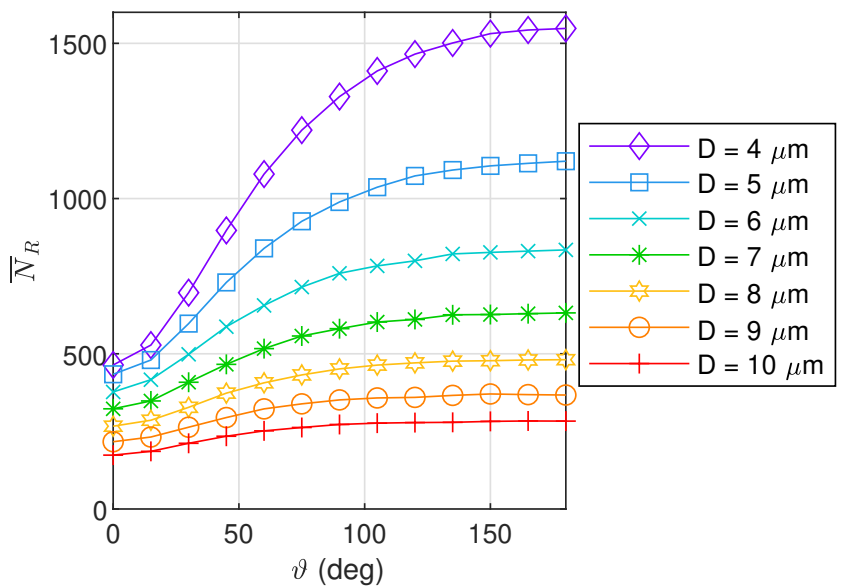

Figure 4: Mean number of absorbed molecules at $R \mathbf{X}_{\mathrm{T}}$ versus $\vartheta$, for $R=1 \mu \mathrm{m}$.

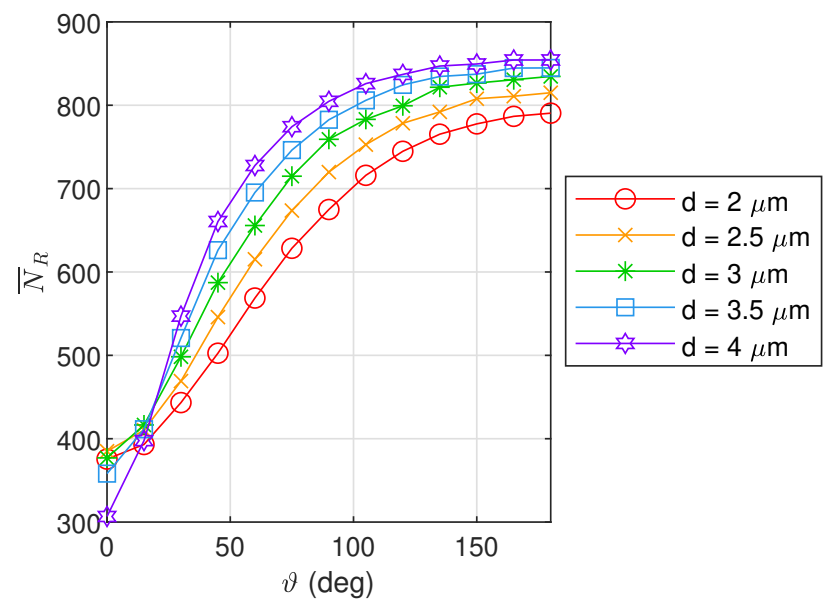

Figure 5: Mean number of absorbed molecules at $R \mathbf{X}_{\mathrm{T}}$ versus $\vartheta$, for $D=6 \mu \mathrm{m}$.

$\mathrm{RX}_{\mathrm{I}}$ is greatly reduced, yet not completely negligible, especially for low values of $D$.

\subsection{Mean number of absorbed molecules versus $d$ for fixed $D$}

In the previous two subsections $d$ was chosen as tightly coupled with $D$. Here we release such a constraint to investigate the behavior of the system when only the distance between the two receivers varies. Therefore, we chose $D=6 \mu \mathrm{m}$, a trade-off between having few meaningful values of $d$, due to the possible overlap of the cells, and having a large set of low sensitive (on $\bar{N}_{R}$ ) values of the same quantity.

The mean number of absorbed molecules for $D=6 \mu \mathrm{m}$ is shown in Fig. 5. For $\vartheta=0^{\circ}$, the minimum number of molecules absorbed at $\mathrm{RX}_{\mathrm{T}}$ occurs when $d=4 \mu \mathrm{m}$ : the lower is $d$, the higher is $\bar{N}_{R}$. For 


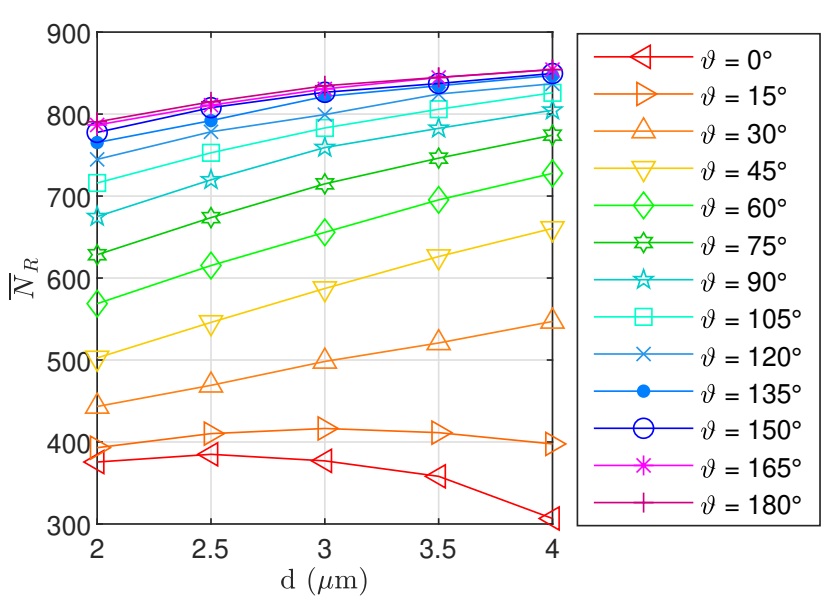

Figure 6: Mean number of absorbed molecules at $\mathbf{R} \mathbf{X}_{\mathrm{T}}$ versus $d$, for $D=6 \mu \mathrm{m}$.

$\vartheta>15^{\circ}$ the situation reverses and recalls the previous analysis: the higher is $d$, the higher is $\bar{N}_{R}$. Thus, we can split the system behavior into two regions. In the first one $\left(\vartheta \leq 15^{\circ}\right)$ it is likely prevailing the "blocking" effect of the interfering cell on the transmitter, which is more evident for $\mathrm{RX}_{\mathrm{I}}$ closer to TX. In the second region, the number of absorbed molecules reduces for smaller $d$, where the dominant effect is the "shadowing" of $\mathrm{RX}_{\mathrm{I}}$ on $\mathrm{RX}_{\mathrm{T}}$.

The $\bar{N}_{R^{-}} d$ plot (Fig. 6) highlights that the number of absorbed molecules is not monotonically increasing for $\vartheta \leq 15^{\circ}$, but a threshold approach in angle estimation is still possible. The analysis of these results confirms that $d=D / 2$ is a suitable choice to represent the angle estimation problem and, therefore, it will be adopted in the following.

\subsection{Angle estimation}

Finally, we performed the angle estimation for the selected hypotheses $d=D / 2$ and $R=1 \mu \mathrm{m}$. The measured number of absorbed molecules for each experiment is compared to the expected tabulated value intervals and the corresponding angle is taken as estimate $\hat{\vartheta}$. The mean-squared error (MSE) is adopted as figure of merit

$$
\operatorname{MSE}=\frac{1}{M} \sum_{i=1}^{M}\left(\hat{\vartheta}_{i}-\vartheta\right)^{2},
$$

which gives the variance of the error. The resulting standard deviation for $M=1000$ is shown in Fig. 7 along with $\vartheta$. We can observe that:

- the MSE always increases with $D$ (and so, $d$ ). The higher is $D$, the lower is the number of received molecules at the target receiving cell. This turns into tighter thresholds, i.e. into a higher probability to select the adjacent angle bins. From another point of view, the effect of the interferer on the target is less exploitable for the purposes of estimating the relative angle under which it is seen by the receiver with respect to transmitter;

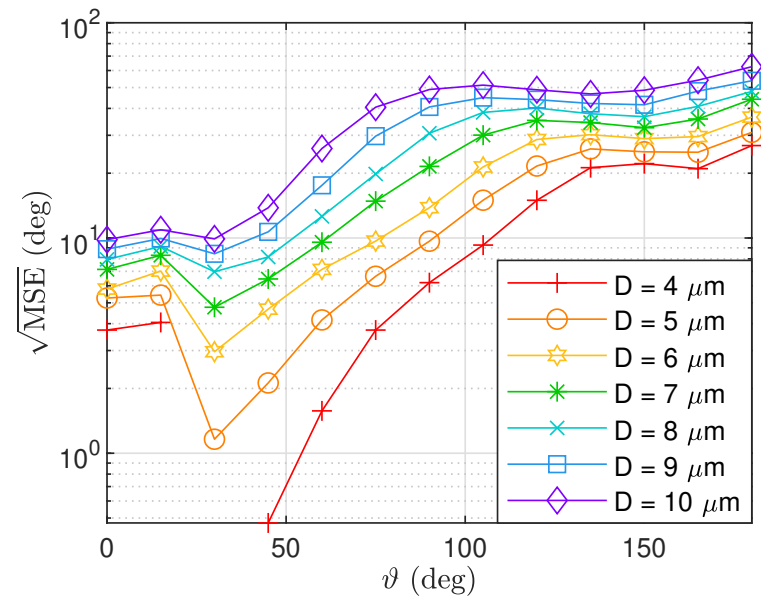

Figure 7: Mean-squared error along with the estimated angles values, for different choices of $D(\mu m)$, and $d=D / 2$.

- the lowest MSE is obtained for $30^{\circ}$, and the jump from $15^{\circ}$ to $30^{\circ}$ is higher when $D$ is lower. The relative angle $\vartheta=30^{\circ}$ is the best compromise for angle estimation, i.e. between the disturbing effect of an interferer near to the transmitter and that near to the target receiver;

- there is an increase in the MSE for $\vartheta>30^{\circ}$ followed by a bending in the range $90^{\circ}-165^{\circ}$;

- the angle estimation for $\vartheta=180^{\circ}$ is always the worst case. This was expected, since for large distances from $\mathrm{RX}_{\mathrm{I}}$ the number of received molecules is the lowest.

\section{PROPOSED ANALYTICAL MODELS}

The estimate of the relative angle between the two absorbing cells would be faster and easier if we had an analytical model of the MC channel impulse response in presence of an absorbing interferer. This would save the simulation time needed to set the intervals that identify each angle estimate for each scenario. In [16], for one single, spherical, fully absorbing receiver of radius $R$ and a pointwise transmitter at distance $D$, the cumulative absorbing probability is derived as

$$
F(D, t)=\frac{R}{D} \operatorname{erfc}\left(\frac{D-R}{\sqrt{4 c_{d} t}}\right),
$$

and the cumulative amount of absorbed molecules can be computed as $N_{T} F(D, t)$. The channel impulse response is obtained by taking the derivative of (4) with respect to $t$, which reads

$$
f(D, t)=\frac{R}{D} \frac{D-R}{\sqrt{4 \pi D t^{3}}} e^{-\frac{(D-R)^{2}}{4 c_{d} t}} .
$$

In [2], the authors empirically observe that the effect of an interfering cell can be accounted for simply as a reduction of the number of molecules $N_{T}$ emitted by the transmitter. The cumulative absorbing probability (4) still holds, provided that $N_{T}$ is reduced by a scaling factor $I$ that is a function of the distance between the transmitter and the interfering cell and of the relative angle between the two absorbing cells. An empirical law is defined where the coefficients used in the function are inferred by using the Levenberg-Marquardt 


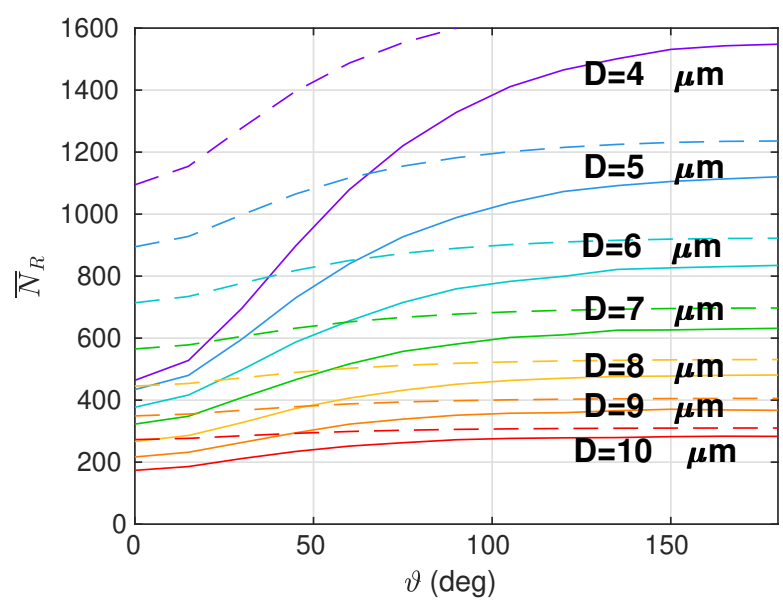

Figure 8: Mean number of absorbed molecules at $\mathbf{R} \mathbf{X}_{\mathrm{T}}$ versus $\vartheta$, for $R=1 \mu \mathrm{m}$. Comparison of simulations (solid curves) and proposed model (dashed curves) of (6).

algorithm to fit simulation results. Unfortunately, these coefficients depend on the receiver to transmitter distance and on the relative angle between the two receivers. Therefore, simulation is still needed to tune the coefficients for each scenario.

To overcome this drawback, we simplify the analytical model that takes into account the mutual reciprocal interference. Firstly, we keep from [2] the idea to model the presence of the interfering cell by counting the number of molecules that are subtracted from the environment, but we consider this mutual effect between the two absorbing cells. For each cell, we multiply the cumulative absorption probability (4) by an amount of molecules that is the emitted $N_{T}$ progressively reduced by the other absorbing cell. This leads to the following system of equations, named as 'model A', to describe the cumulative number of molecules absorbed in each time instant

$$
\left\{\begin{array}{l}
N_{R}(t)=\left(N_{T}-N_{I}(t)\right) F(D, t), \\
N_{I}(t)=\left(N_{T}-N_{R}(t)\right) F\left(d_{I}, t\right),
\end{array}\right.
$$

where $d_{I}$ is the distance between the transmitter and the interfering cell. In Fig. 8 we compare the simulation results with the predictions of model A. Albeit for large $\vartheta$ values simulation and model fairly agree, model A fails to capture the effect of the reciprocal position of the receivers, which is accounted for in [2] by adjusting the scaling factor $I$ as a function of such position. On the contrary, in (6) the expressions of $N_{R}(t)$ and $N_{I}(t)$ depend solely on the distances of the two cells from the transmitter and not on the relative distance between them. Thus, the angle $\vartheta$ influences $N_{R}(t)$ by affecting the distance $d_{I}$ only.

In order to take into account the relative distance between the two receivers, a second model here proposed considers each absorbing cell as a source of negative molecules for the other, since its effect is to subtract molecules from the environment. This model, referred in the following as 'model B', computes the number of molecules absorbed by each receiver per unit time $n_{R}(t)$ and $n_{I}(t)$

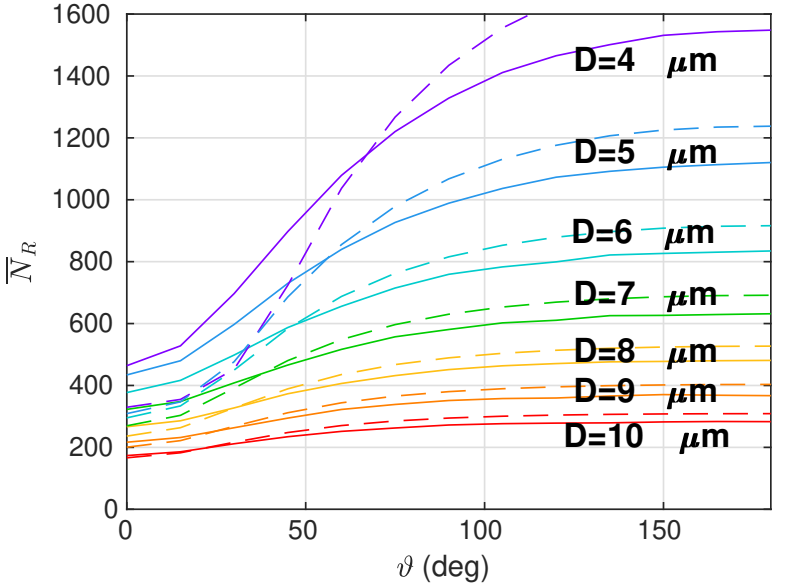

Figure 9: Mean number of absorbed molecules at $R \mathbf{X}_{\mathrm{T}}$ versus $\vartheta$, for $R=1 \mu \mathrm{m}$. Comparison of simulations (solid curves) and proposed model (dashed curves) of (7).

by numerically solving the system of equations

$$
\left\{\begin{array}{l}
n_{R}(t)=N_{T} f(D, t)-n_{I}(t) * f(d, t), \\
n_{I}(t)=N_{T} f\left(d_{I}, t\right)-n_{R}(t) * f(d, t),
\end{array}\right.
$$

where $*$ denotes the convolution and $f(d, t)$ is the channel impulse response (5). $N_{R}(t)$ and $N_{I}(t)$ can be computed by integrating $n_{R}(t)$ and $n_{I}(t)$ in time.

The comparison with the simulation case of Fig. 4 is given in Fig. 9. Model B clearly captures better than model A the interaction between the two cells, in particular for large values of the distance $D$ (a light, systematic overestimate by model B of the number of absorbed molecules is still under investigation). For low values of $D$ and small angles $\vartheta$ some refinements are still needed, but the agreement is encouraging and a deepening is ongoing. Simulation data could also be used to identify the angle intervals for small values of the distance $D$ for which the model predictions do not seem to be accurate, saving simulation time for the scenarios with large values of $D$.

\section{CONCLUSION AND FUTURE WORK}

In this work we addressed the problem of localizing an interferer that is present in the same environment of a diffusive molecular communication system defined by one transmitting and one receiving cell. In particular, we exploited the perturbation introduced by an absorbing interferer on the amount of molecules absorbed by the receiving cell to propose an approach to estimate the relative angle of the interfering cell with respect to the segment that joins transmitter and receiver. The search of a suitable scenario accounted for different values of:

- distance between the transmitting and the receiving cell;

- distance between the interfering and the receiving cell;

- radius of the interfering and of the receiving cells.

The selected scenario was used to perform the relative angle estimation. 
Simulations were conducted to validate our proposed method. The obtained results show a non-trivial behavior of the molecular communication system. Not all the positions of the interferer are estimated with the same accuracy: in general, the standard deviation of the error increases with the distance between transmitter and receiver and with the relative angle. On the other hand, for small angles and small distances, the conjecture is that the "blocking" effect on the transmitted molecules becomes so relevant that the angle estimation becomes harder, due to a small variation in the number of absorbed molecules. The consequence is that the highest accuracy is observed for a slightly larger relative angle, i.e. around $30^{\circ}$. Finally, in order to save simulation time, we also proposed two different analytical models that do not require parameter tuning to predict the effect of the interfering cell on the receiver. The latter, namely model B, shows a fair agreement with simulation data, in particular for large values of the receiver to transmitter distance $D$. A refinement of the proposed model is one of the objects of our future work, in particular for low values of $D$ when its accuracy is lower.

The presented results, obtained with some simplifying yet consistent hypotheses, encourage us to further investigate the considered scenario. Possible extensions are a study on the sensitivity of the angle estimation to different amounts of released molecules or to more general distances among transmitter, interferer, and receiver. Furthermore, a more realistic scenario may be introduced. The transmitter might be modeled as a finite dimension cell, releasing the molecules from the cell gap junctions on the membrane. The number of receiving and transmitting cells could be more than two, like a real bacterial colony, and with different dimensions. Additional source of interference could arise from different cells, such in the case of different bacterial species. This source of disturbance has a relevant effect from a biological point of view, since the information content encoded by the molecules is uselessly dispersed. Finally, the static environment and the fully absorbing receiver hypotheses could be relaxed as well.

\section{REFERENCES}

[1] Bruce Alberts, Dennis Bray, Karen Hopkin, Alexander Johnson, Julian Lewis, Martin Raff, Keith Roberts, and Peter Walter. 2014. Essential Cell Biology (4th ed.).

[2] Xu Bao, Jie Lin, and Wence Zhang. 2019. Channel modeling of molecular communication via diffusion with multiple absorbing receivers. IEEE Wireless Communications Letters 8, 3 (2019), 809-812.

[3] Luca Felicetti, Mauro Femminella, Gianluca Reali, and Pietro Liò. 2016. Applications of molecular communications to medicine: A survey. Nano Communication Networks 7 (2016), 27-45.

[4] Bruce I Henry, Trevor AM Langlands, and Peter Straka. 2010. An introduction to fractional diffusion. In Complex Physical, Biophysical and Econophysical Systems. World Scientific, 37-89.

[5] Jiun-Ting Huang, Hsin-Yu Lai, Yen-Chi Lee, Chia-Han Lee, and Ping-Cheng Yeh. 2013. Distance estimation in concentration-based molecular communications. In 2013 IEEE Global Communications Conference (GLOBECOM). IEEE, 2587-2591.

[6] Kok-Fai Kong, Cuong Vuong, and Michael Otto. 2006. Staphylococcus quorum sensing in biofilm formation and infection. International journal of medical microbiology 296, 2-3 (2006), 133-139.

[7] Bon-Hong Koo, Changmin Lee, H Birkan Yilmaz, Nariman Farsad, Andrew Eckford, and Chan-Byoung Chae. 2016. Molecular MIMO: From theory to prototype. IEEE Journal on Selected Areas in Communications 34, 3 (2016), 600-614.

[8] Changmin Lee, H Birkan Yilmaz, Chan-Byoung Chae, Nariman Farsad, and Andrea Goldsmith. 2017. Machine learning based channel modeling for molecular MIMO communications. In 2017 IEEE 18th International Workshop on Signal Processing Advances in Wireless Communications (SPAWC). IEEE, 1-5.

[9] Yi Lu, Matthew D. Higgins, Adam Noel, Mark S. Leeson, and Yunfei Chen. 2016. The effect of two receivers on broadcast molecular communication systems. IEEE
Transactions on Nanobioscience 15, 8 (Dec. 2016), 891-900.

[10] Adam Noel, Karen C Cheung, and Robert Schober. 2014. Bounds on distance estimation via diffusive molecular communication. In 2014 IEEE Global Communications Conference. IEEE, 2813-2819.

[11] Song Qiu, Weisi Guo, Bin Li, Yue Wu, Xiaoli Chu, Siyi Wang, and Yin Yao Dong. 2015. Long range and long duration underwater localization using molecular messaging. IEEE Transactions on Molecular, Biological, and Multi-scale Communications 1,4 (Dec. 2015), 363-370.

[12] David Ribet and Pascale Cossart. 2015. How bacterial pathogens colonize their hosts and invade deeper tissues. Microbes and infection 17, 3 (2015), 173-183.

[13] Jitesh A Soares and Brian MM Ahmer. 2011. Detection of acyl-homoserine lactones by Escherichia and Salmonella. Current opinion in microbiology 14, 2 (2011), 188-193.

[14] Gerard J. Tortora, Berdell R. Funke, and Christine L. Case. 2010. Microbiology: an Introduction. Pearson Benjamin Cummings Publishing Company, inc., Redwood City, California.

[15] Xiayang Wang, Matthew D Higgins, and Mark S Leeson. 2015. Distance estimation schemes for diffusion based molecular communication systems. IEEE Communications Letters 19, 3 (2015), 399-402.

[16] H Birkan Yilmaz, Akif Cem Heren, Tuna Tugcu, and Chan-Byoung Chae. 2014. Three-dimensional channel characteristics for molecular communications with an absorbing receiver. IEEE Communications Letters 18, 6 (2014), 929-932. 